\title{
Laser-Light Scattering from Ringed Spherulites in Poly(decamethylene terephthalate): Observation and Interpretation of Unusual "Ring- Scattering" Patterns
}

\author{
Irena DANIEwSKA, Takeji HASHIMOTo, ${ }^{*}$ and Akemi NAKAI* \\ Department of Chemistry, Technical University (Politechnika), \\ Koszykowa 75, 00-662, Warsaw, Poland \\ * Department of Polymer Chemistry, Faculty of Engineering, \\ Kyoto University, Kyoto, Japan
}

(Received October 25, 1983)

\begin{abstract}
The morphology of crystallized poly(decamethylene terephthalate) samples was studied by optical microscopy and the small-angle light scattering method. Ringed spherulites exhibiting unusual $H_{\mathrm{v}}$ and $V_{\mathrm{v}}$ scattering patterns were observed and found to be formed at crystallization temperatures in the range from 0 to $80^{\circ} \mathrm{C}$. The observed scattering patterns of ringed spherulites were analyzed and compared with the published results. It is proposed that the observed unusual scattering patterns may have resulted from light scattering from ringed spherulites with the optical axis making an angle of $45^{\circ}$ to the spherulite radius.
\end{abstract}
KEY WORDS
Poly(decamethylene terephthalate)
Morphology / Ringed Spherulite / Light Scattering /
Crystallization

The most typical forms obtained for a crystalline polymer structure by melt crystallization are spherulites. Morphological studies, using mainly microscopic and light scattering methods, revealed various types of spherulites depending on crystallization conditions. ${ }^{1}$ Thus, the following types of spherulites were reported: $0-90^{\circ}$ radial and $0-90^{\circ}$ ringed spherulites with the principal polarizability axis inclined at 0 or $90^{\circ}$ to the spherulite radius, ${ }^{2-4}$ and $45^{\circ}$ radial spherulites with the principal polarizability axis inclined at $45^{\circ}$ to the spherulite radius. $^{4-6}$ Although ringed spherulites of the $45^{\circ}$-type were predicted theoretically, ${ }^{4,5,31}$ none of their scattering patterns have so far been reported in the literature.

In the present work, unusual scattering patterns due to ringed spherulites observed in poly(decamethylene terephthalate) are discussed. We suggest that these patterns result from ringed spherulites with the optical axis inclined at about $45^{\circ}$ to the spherulite radius.

\section{EXPERIMENTAL}

Poly(decamethylene terephthalate) designated 10GT, prepared from dimethyl terephthalate and decamethylene glycol according to the procedure described earlier, ${ }^{7}$ were investigated. The polymer was characterized by the number-average molecular weight, $M_{n}$, determined from the content of hydroxyl end groups, and by the equilibrium melting temperature, $T_{\mathrm{m}}$, measured under an optical microscope with a heated stage. The temperature of maximum crystallization rate, $T_{\max }, 8,9$ and the glass transition temperature, $T_{\mathrm{g}},{ }^{10,11}$ were calculated using the following relationships:

$$
T_{\max }=(0.82-0.85) T_{\mathrm{m}}
$$




$$
T_{\mathrm{g}}=(0.66-0.67) T_{\mathrm{m}}
$$

Samples for morphological investigations were prepared as follows. A small amount of the polymer was melted between microscope cover-strips and heated for $5 \mathrm{~min}$ at a temperature $15^{\circ} \mathrm{C}$ above $T_{\mathrm{m}}$. The sample was then placed in a thermostat and kept there for at least $20 \mathrm{~h}$ and annealed at intervals of $10^{\circ} \mathrm{C}$ in the range from -10 to $100^{\circ} \mathrm{C}$. In this way, a set of 12 samples crystallized at different temperatures were obtained.

The morphology of each sample was studied by otpical microscopy and the small-angle light scattering (SALS) method. The scattering intensity was measured to determine spherulite radii and ring spacings. The average spherulite radii were calculated from the clover-leaf scattering pattern using the formula of Stein and Rhodes: ${ }^{12}$

$$
U=\left(\frac{4 \pi R}{\lambda}\right) \sin \frac{\theta}{2}
$$

where $R$ is the spherulite radius, $\lambda$, the light wavelength, and $\theta$, the scattering angle. The parameter $U$ is 4 at the angle of maximum scattering intensity $\left(\theta=\theta_{\max }\right)$ along one of the clover-leaves.

The "ring spacing" (the spacing between successive dark or white fringes of the banded spherulites observed under a polarized light microscope) was calculated from the positions of the ring scattering maxima, i.e., the scattering maxima attributable to ring spacings. As shown in Figure 2, in some cases, three ring scattering maxima designated as I to III appeared at the scattering angles $\theta_{m, 1}$ to $\theta_{m, 3}$. The apparent ring spacing $\rho_{\mathrm{I}}, \rho_{\mathrm{II}}$, and $\rho_{\mathrm{III}}$ were estimated using the following relationship:

$$
\lambda=2 \rho_{\mathrm{i}} \sin \left(\theta_{m, i} / 2\right) \quad(i=\mathrm{I}, \mathrm{II}, \mathrm{III})
$$

Usually, the ring spacing is equal to one-half the distance for one complete rotation of the optical axis along the spherulite radius. ${ }^{12}$ However, in this particular case, a further theoretical interpretation was needed to ex- tract physical significance from the ring spacings $\rho_{i}$ 's.

\section{RESULTS AND DISCUSSION}

The characteristics of the original 10GT sample are shown in Table I. The influence of the crystallization temperature on the morphology of this sample is shown in Figure 1 (optical micrographs) and Figure $3\left(H_{\mathrm{v}}\right.$ and $V_{\mathrm{v}}$ scattering patterns).

The crystallization temperature affected the morphology of the 10GT sample considerably. Near the glass temperature, i.e., at crystallization temperatures $T_{\text {cr }}<0^{\circ} \mathrm{C}$, very small crystalline superstructures were found (Figure 1a). Light scattering studies showed that these structures were of the ringed spherulite type (Figure 3a). At crystallization temperatures $0 \leqslant T_{\mathrm{cr}} \leqslant 80^{\circ} \mathrm{C}$, ringed spherulites of various sizes were observed (Figures $1 \mathrm{~b}-\mathrm{d}$ ). Near the melting point, $T_{\mathrm{cr}} \geqslant 90^{\circ} \mathrm{C}$, the plate-like spherulites were found (Figure 1e). The scattering patterns (Figure 3d) showed the last spherulites to be poorly defined and nonringed.

The scattering patterns due to ringed spherulites consisted of two types of scattering patterns: low-angle pattern (clover-leaves under $H_{\mathrm{v}}$ ) and wide-angle arcs (Figures $3 \mathrm{a}, \mathrm{b}, \mathrm{c})$. Figure 2 shows the schematic diagrams of these patterns. What is unusual is the azimuthal position of arcs I. On the other hand, arcs II and III are in agreement with the literature data for ringed spherulites. ${ }^{13,14,26,29}$ The low-angle patterns were qualitatively identical at all crystallization temperatures, and some differences were observed in the wide-angle patterns; i.e. the number of arcs

\begin{tabular}{|c|c|c|c|}
\hline$M_{n}$ & $T_{\mathrm{m}}$ & $T_{\max }$ & $T_{\mathrm{g}}$ \\
\hline $\mathrm{g} \mathrm{mol}^{-1}$ & ${ }^{\circ} \mathrm{C}$ & ${ }^{\circ} \mathrm{C}$ & ${ }^{\circ} \mathrm{C}$ \\
\hline 5700 & 119 & $47-60$ & $-13--15$ \\
\hline
\end{tabular}
and their azimuthal positions were different

Table I. Characteristics of the 10GT sample 
Light Scattering and Crystallization of Poly(decamethylene terephthalate)

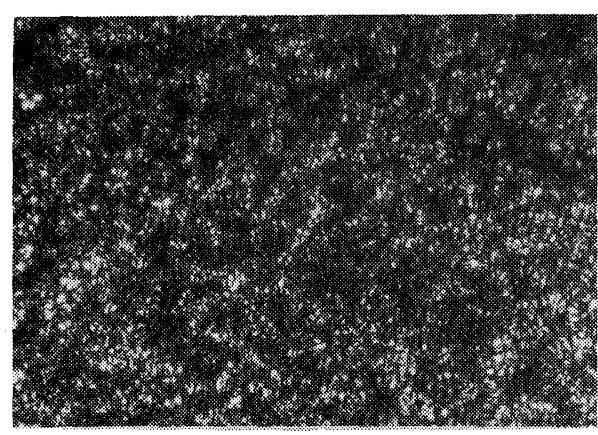

a

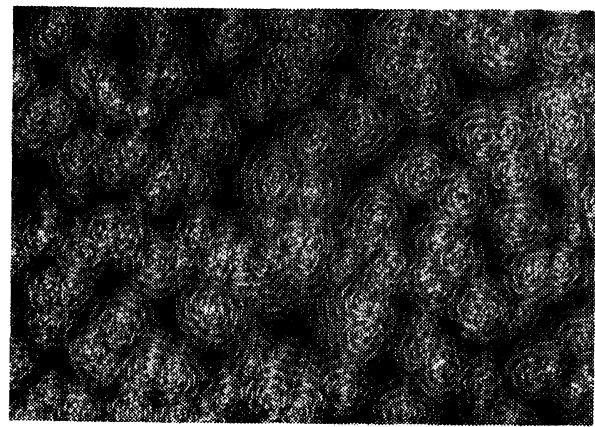

C

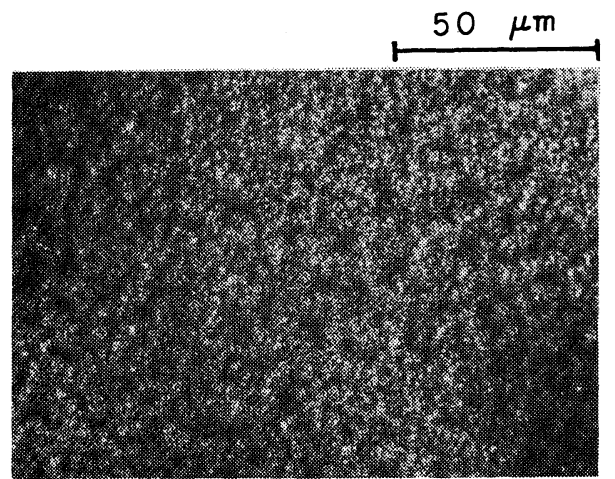

b

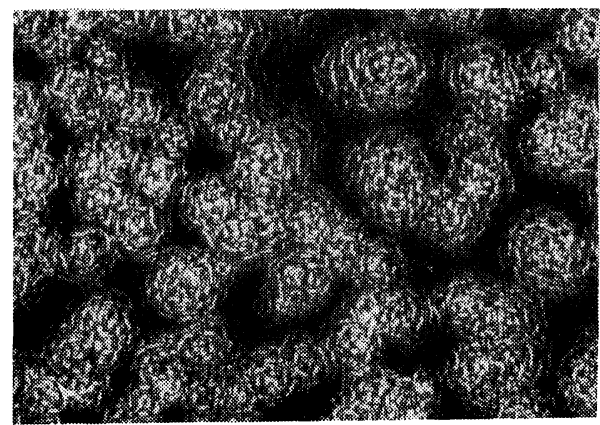

d

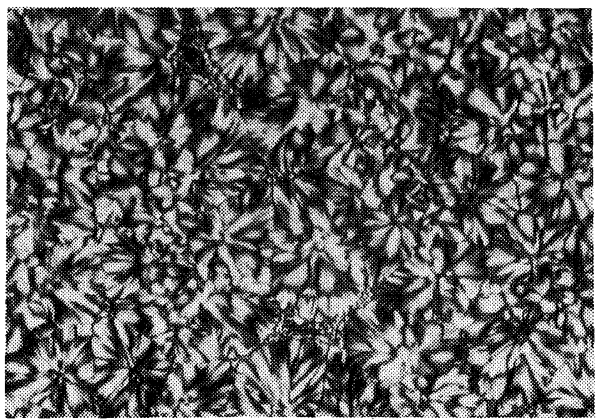

e

Figure 1. Typical micrographs of the $10 \mathrm{GT}$ sample crystallized at various temperarures: $\mathrm{a}$, from -10 to $-5^{\circ} \mathrm{C}$, b, from 0 to $40^{\circ} \mathrm{C}$, c, from 50 to $70^{\circ} \mathrm{C}$; d, $80^{\circ} \mathrm{C}$; e, from 90 to $100^{\circ} \mathrm{C}$.

depending on the crystallization temperature. The samples which crystallized at $-10 \leqslant$ $T_{\mathrm{c}} \leqslant 40^{\circ} \mathrm{C}$ exhibited an $H_{\mathrm{v}}$ scattering pattern containing, in its wide-angle part, only one set of arcs (i.e., the arcs I in Figure 2 and Figure 3a) at the $0-90^{\circ}$ position, i.e., parallel to the polarizer and analyzer axes. These arcs in the $V_{\mathrm{v}}$ scattering pattern shifted to odd mul- 
tiples of $\mu=45^{\circ}$ relative to the polarizer or analyzer axis. The wide-angle $H_{\mathrm{v}}$ scattering pattern of the sample crystallized at 50 to $70^{\circ} \mathrm{C}$ consisted of two sets of arcs, i.e., four arcs parallel to the polarizer and analyzer axes (arcs I) and four arcs at odd multiples of $\mu=45^{\circ}$

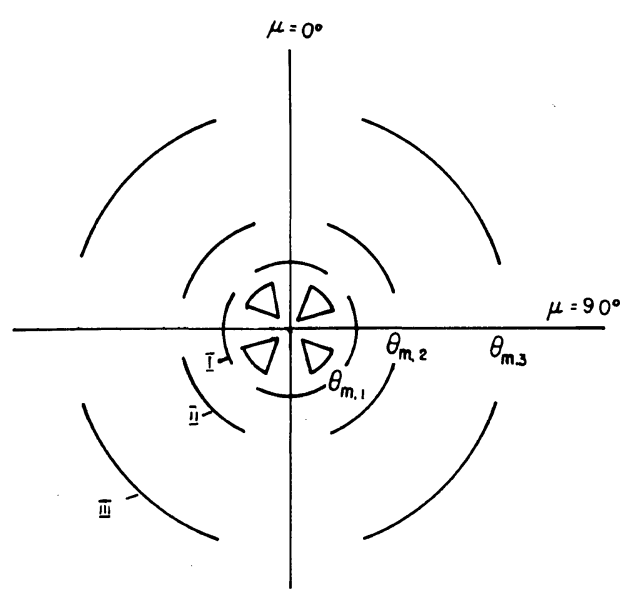

a

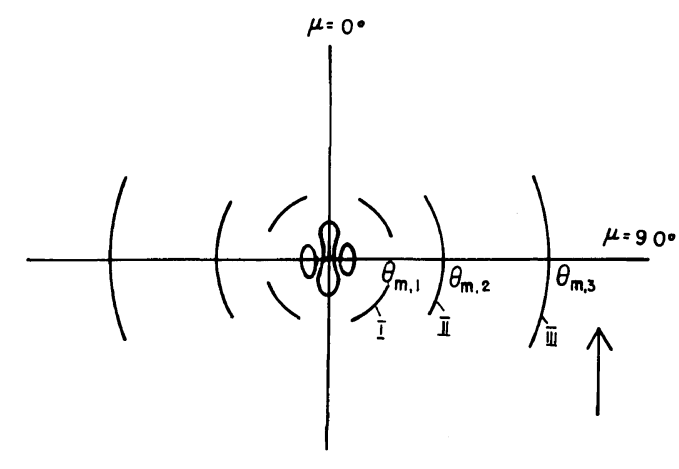

b

Figure 2. A schematic diagram of (a) $H_{\mathrm{v}}$ and (b) $V_{\mathrm{v}}$ scattering patterns.

$H_{V}$

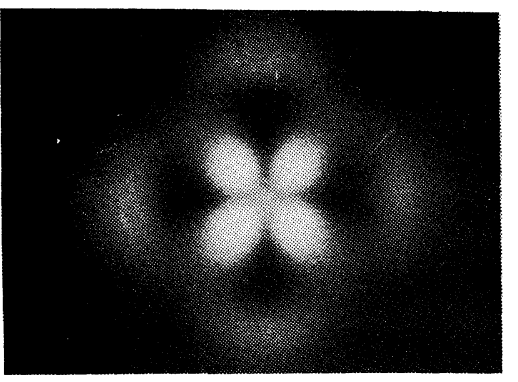

b

a

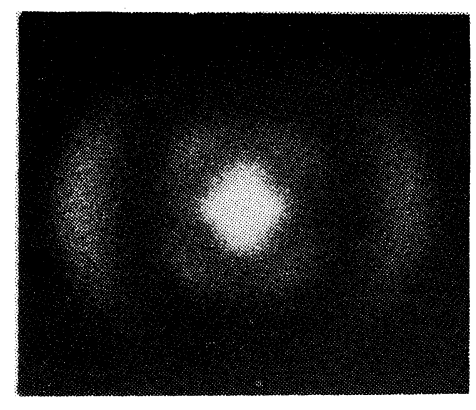

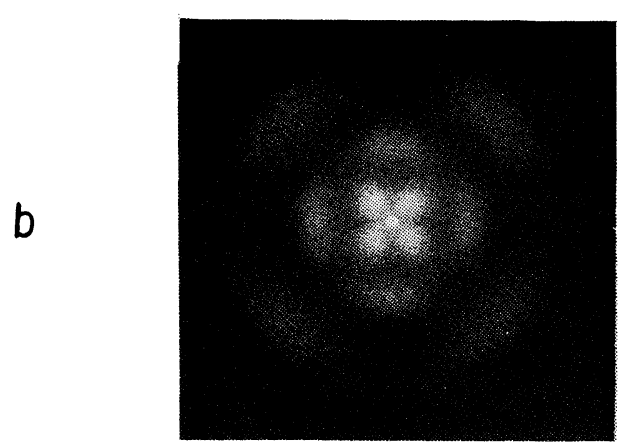

Fig. 3

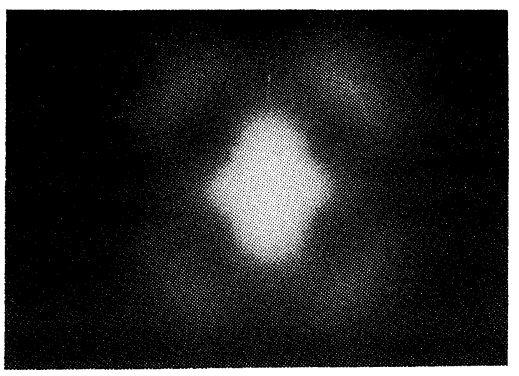

$V_{V}$ 
Light Scattering and Crystallization of Poly(decamethylene terephthalate)

Hv
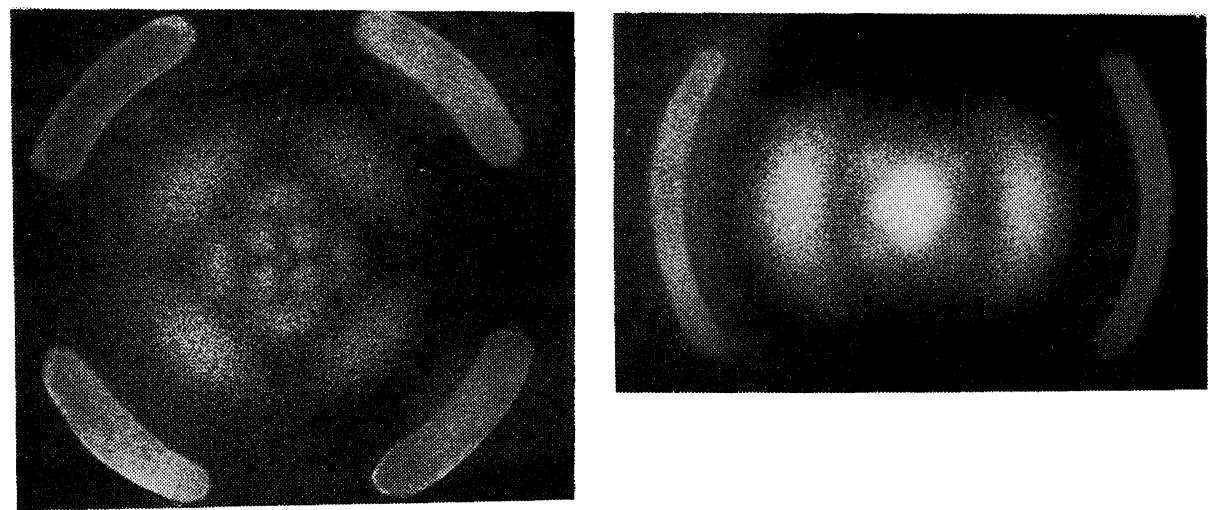

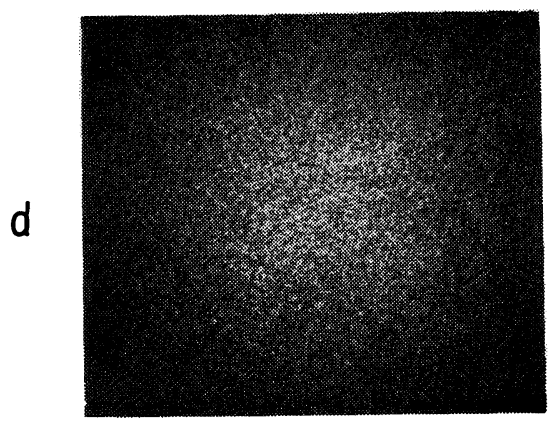

Figure 3. Typical scattering patterns of the $10 \mathrm{GT}$ sample crystallized at various temperatures: a, from -10 to $40^{\circ} \mathrm{C}$; b, from 50 to $70^{\circ} \mathrm{C} ; \mathrm{c}, 80^{\circ} \mathrm{C}$; d, from 90 to $100^{\circ} \mathrm{C}$.

relative to the polarizer and analyzer axes (arcs II) (see Figures $3 \mathrm{~b}$ and c). The arcs $\mathrm{I}$ in the $V_{\mathrm{v}}$ scattering pattern shifted to odd multiples of $\mu=45^{\circ}$, while the four arcs II from the $H_{\mathrm{v}}$ pattern corresponded to two arcs in the $V_{\mathrm{v}}$ pattern at $90^{\circ}$ relative to the direction of light polarization. The $H_{\mathrm{v}}$ scattering pattern of the sample crystallized at $80^{\circ} \mathrm{C}$ (Figure 3c) was similar to those shown in Figure $3 \mathrm{~b}$; however, besides the arcs I at $0-90^{\circ}$, two other sets, arcs II and III, were observed at odd multiples of $\mu=45^{\circ}$. The intensity of the arcs III was so weak as to preclude recording them on the picture, and were thus added to the picture as drawings. Arcs II and III in the $V_{\mathrm{v}}$ patterns shifted to the position at $90^{\circ}$ relative to the direction of light polarization. The scattering
Table II. Spherulite characteristics

\begin{tabular}{|c|c|c|c|c|}
\hline \multirow{3}{*}{$\begin{array}{c}\begin{array}{c}\text { Crystal- } \\
\text { lization } \\
\text { temperature }\end{array} \\
{ }^{\circ} \mathrm{C}\end{array}$} & \multirow{3}{*}{$\begin{array}{c}\begin{array}{c}\text { Spherulite } \\
\text { radius, } R\end{array} \\
\mu \mathrm{m}\end{array}$} & \multicolumn{3}{|c|}{ Ring spacing } \\
\hline & & \multirow{2}{*}{$\begin{array}{c}\text { Arcs } \\
\mathrm{I} \cdot \rho_{\mathrm{I}}\end{array}$} & \multirow{2}{*}{$\begin{array}{c}\text { Arcs } \\
\mathrm{II} \cdot \rho_{\mathrm{II}}\end{array}$} & \multirow{2}{*}{$\begin{array}{c}\text { Arcs } \\
\mathrm{III} \cdot \rho_{\mathrm{III}} \\
\mu \mathrm{m}\end{array}$} \\
\hline & & & & \\
\hline-10 & 1.3 & 0.6 & & \\
\hline 0 & 2.2 & 1.3 & & \\
\hline 10 & 2.6 & 1.4 & & \\
\hline 20 & 2.4 & 1.7 & & \\
\hline 30 & 2.7 & 1.6 & & \\
\hline 40 & 3.3 & 1.7 & & \\
\hline 50 & 7.4 & 2.3 & 1.2 & \\
\hline 60 & 8.2 & 2.7 & 1.2 & \\
\hline 70 & 12.0 & 6.5 & 3.1 & \\
\hline 80 & 12.5 & 8.5 & 4.0 & 1.9 \\
\hline
\end{tabular}




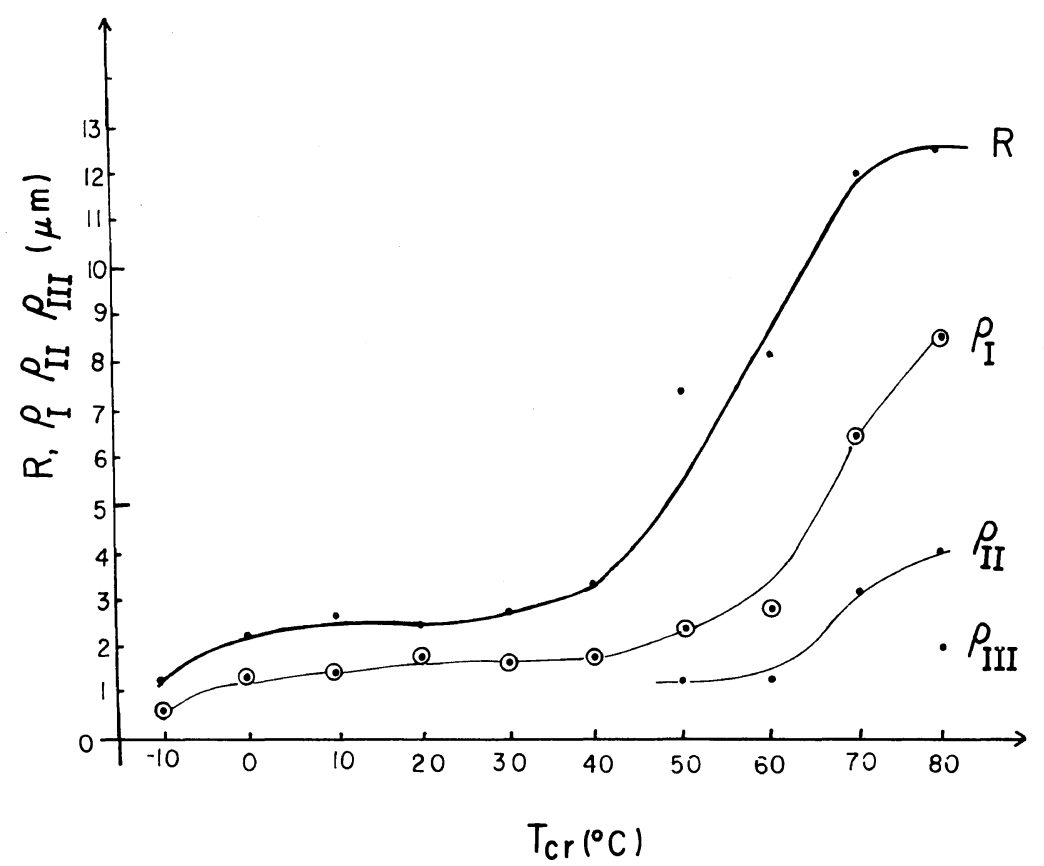

Figure 4. Spherulite radius $(R)$ and ring spacings $\rho_{\mathrm{l}}, \rho_{\mathrm{II}}$, and $\rho_{\mathrm{III}}$ versus crystallization temperature.

patterns of the sample crystallized at 90 to $100^{\circ} \mathrm{C}$ were diffuse with poorly defined clover leaves (Figure 3d), reflecting poorly defined spherulites. Thus, no ringed spherulite was observed in any sample crystallized at these temperatures.

The calculated spherulite radii and ring spacings are shown in Table II and Figure 4. It can be seen that the spherulite radius and ring spacing increase with increasing crystallization temperature, which is consistent with the published results. ${ }^{15,17}$

The azimuthal angular positions of arcs I may be attributed to a certain specific structure of the ringed spherulites not presented fo far in the literature. A detailed analysis of the arcs in scattering patterns should thus be worth while.

We may consider that arcs II form a secondorder reflection of arcs $\mathrm{I}$, since $\rho_{\mathrm{I}}=2 \rho_{\mathrm{II}}$, in accord with the equation for the multipleorder scattering: ${ }^{13,33}$

$$
n \lambda=2 \Lambda \sin \theta_{m, n} / 2 \quad(n=1,2)
$$

where $n$ is the order of reflection and $\Lambda$ is the spacing. This may be questionable, since it has not been shown that the origin of arcs $I$ is identical to that of arcs II and III. We observed that (i) the azimuthal angular position of arcs I and those of arcs II and III relative to the direction of light polarization are different and (ii) the light scattering intensities of arcs I and II are about the same, contrary to the theoretical and experimental conclusions ${ }^{13,18-20}$ that the intensity of the first order reflection is stronger than that of the second order. This last condition is satisfied for arcs II and III, and (iii) $\rho_{\text {I }} \neq 3 \rho_{\text {III }}$, while $\rho_{\text {II }}=2 \rho_{\text {III }}$. Therefore, arcs III may be considered a second-order reflection of arcs II, while arcs I originate from another periodicity. The ring spacings of arcs I, II, and III are summarized in Table II.

The angular dependence of the SALS pattern (clover leaves under $H_{\mathrm{v}}$ ) and of arcs II 
and III for both $H_{\mathrm{v}}$ and $V_{\mathrm{v}}$ patterns indicates that $0-90^{\circ}$ type spherulites are present in the samples. More specificially, the spherulites should be of the $90^{\circ}$-type because of the existence of ring scattering. It should be noted that $0^{\circ}$-type spherulites do not give ringed spherulites. However, arcs $\mathrm{I}$ in both $H_{\mathrm{v}}$ and $V_{\mathrm{v}}$ patterns behave as if they arise from scattering centers with the optical axis inclined at $45^{\circ}$ to the spherulite radius. ${ }^{6,21}$ There are two possible reasons as to why arcs I and II were observed:

(i) different types of spherulites, i.e., $90^{\circ}$ type and $45^{\circ}$-type spherulites coexist in the samples;

(ii) one type of spherulite is present in the samples, and the optical axis of the scattering element is helicoidally rotated about the spherulite radii when the radial fibrils are twisted. Hypothesis (i) should be ruled out. If $90^{\circ}$ - and $45^{\circ}$-type spherulites coexist, the SALS pattern under $H_{\mathrm{v}}$ should contain clover-leaf patterns at both $45^{\circ}$ and $90^{\circ}$ positions, and hence a diffuse halo ${ }^{22}$ or two distinct clover-leaves ${ }^{20,23}$ should be observed. But such patterns were not found in this work. Thus, hypothesis (ii) is discussed in detail in the following section. It should be noted that $\rho_{\mathrm{I}} \cong 2 \rho_{\text {II }}$. If the ring spacing $\rho_{\text {II }}$ corresponds to one-half the twisting pitch of a regular helix, ${ }^{17,24-27}$ the ring spacing $\rho_{\mathrm{I}}$ calculated from arcs I corresponds to the full slip of the lamellae twist.

The temperature of the formation of spherulites discussed is atypical. It is known that the formation of ring spherulites depends on the rate of growth: the known ring spherulites are formed at a slow growth rate near melting temperature, and neither at a fast growth rate near the temperature of the maximum crystallization rate, $T_{\max }$, nor at a slow growth rate near the glass transition temperature. ${ }^{24,28}$ Such dependence was observed for poly(ethylene tetrephthalate), poly(hexamethylene sebacate), poly(hexamethylene adipate) and polyethylene ${ }^{16,29}$ where only ring spherulites were formed during slow crystallization near the melting temperature. In our case, the ring spherulites were formed over wide range of temperature from 80 to $-10^{\circ} \mathrm{C}$, i.e., from near $T_{\max }$ (fast growth rate) to near $T_{\mathrm{g}}$ (slow growth rate). The temperature range of formation of the ring spherulites in our 10GT sample was similar to that observed for 10GT samples of lower molecular weight, ${ }^{7}$ which gave $45^{\circ}$-type radial spherulites. According to Stein, ${ }^{30} 45^{\circ}$ type spherulites are formed if macromolecules fold at $45^{\circ}$ relative to the lamella surface and the lamellae are appropriately situated. It is very likely that such conditions were prevalant in the ringed spherulites of our sample.

\section{Theoretical Interpretation on Unusual Ring-} Scattering Patterns

This section presents a theoretical interpretation of the observed unusual ring-scattering patterns. As discussed in the previous section, our model is based on ringed spherulites with the optical axis inclined at an angle $\beta$ (close to $45^{\circ}$ ) to the spherulite radius (see Figure 5).

The scattering amplitude $E_{\mathrm{Ab}}$ under a given polarization condition $A_{\mathrm{b}}$ is given by the Fourier transform of the spatial distribution of induced dipole moment $(\boldsymbol{M} \cdot \boldsymbol{O})_{\mathrm{Ab}}$ :

$$
E_{\mathrm{Ab}}=\mathscr{F}\left\{(\boldsymbol{M} \cdot \boldsymbol{O})_{\mathrm{Ab}}\right\}
$$

where $\mathscr{F}(X)$ designates the Fourier transform of $X(\boldsymbol{r})$. Our discussion can be simplified without sacrificing physical significance by con-

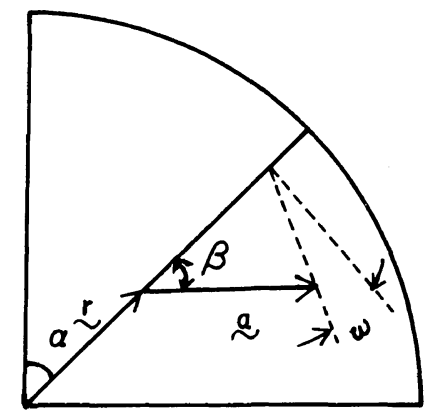

Figure 5. Model of a two-dimensional spherulite with an optical axis a inclined at a constant polar angle $\beta$ but helicoidally rotated about the radial vector $\mathbf{r}$ of the spherulite. 
sidering the scattering from a two-dimensional ringed spherulite (see Figure 5). The vector $\boldsymbol{a}$ is a unit vector parallel to the optical axis. The scattering elements are assumed to have uniaxial anisotropy with polarizabilities $\alpha_{1}$ and $\alpha_{2}$ parallel and perpendicular to $\boldsymbol{a}$, respectively. The vector $\boldsymbol{a}$ is rotated helicoidally about the radial vector $\boldsymbol{r}$ of a spherulite with a constant polar angle $\beta, \omega$ being the rotational angle which depends on $\boldsymbol{r}$.

At small scattering angles, the induced dipole moments $H_{\mathrm{v}}$ and $V_{\mathrm{v}}$ scattering conditions are given $b^{4,31}$

$$
\begin{aligned}
(\boldsymbol{M} \cdot \boldsymbol{O})_{H_{v}}= & \frac{1}{2} E_{0}\left(\alpha_{1}-\alpha_{2}\right)\left[\left(\cos ^{2} \beta\right.\right. \\
& \left.-\sin ^{2} \beta \cos ^{2} \omega\right) \sin 2 \alpha \\
& -\sin 2 \beta \cos \omega \cos 2 \alpha]
\end{aligned}
$$

and

$$
\begin{aligned}
(\boldsymbol{M} \cdot \boldsymbol{O})_{V_{\mathrm{v}}}= & E_{0}\left(\alpha_{1}-\alpha_{2}\right)\left[\cos ^{2} \alpha \cos ^{2} \beta\right. \\
& +\sin ^{2} \beta \sin ^{2} \alpha \cos ^{2} \omega \\
& \left.+\frac{1}{2} \sin 2 \alpha \sin 2 \beta \cos \omega\right]+E_{0} \alpha_{2}
\end{aligned}
$$

where $E_{0}$ is the electric field strength of the incident light. It should be noted that eq 4 and 5 satisfy the physical indistinguishability of the optical axis orientations $\boldsymbol{a}$ and $-\boldsymbol{a}$ because $(\boldsymbol{M} \cdot \boldsymbol{O})_{H_{\mathrm{v}}}$ and $(\boldsymbol{M} \cdot \boldsymbol{O})_{V_{\mathrm{v}}}$ are invariant with the replacement of $\beta$ by $\beta+\pi$. For homogeneous spherulites, $\beta,\left(\alpha_{1}-\alpha_{2}\right)$, and $\alpha_{2}$ are constant. It was assumed that $\omega$ varies linearly with $r$, so that

$$
\omega=2 \pi(l / R) r
$$

where $R$ is the spherulite radius and $l$ is the number of turns of the optical axis about the radial distance $R^{4,12,31}$

The simplest case of $\beta=90^{\circ}$ is considered first where,

$$
(\boldsymbol{M} \cdot \boldsymbol{O})_{H_{\mathrm{v}}}=-\frac{1}{2} E_{0}\left(\alpha_{1}-\alpha_{2}\right) \cos ^{2} \omega \sin 2 \alpha
$$

$$
(\boldsymbol{M} \cdot \boldsymbol{O})_{V_{\mathrm{v}}}=E_{0}\left(\alpha_{1}-\alpha_{2}\right) \sin ^{2} \alpha \cos ^{2} \omega+E_{0} \alpha_{2}
$$

Owing to the rotation of the optical axis according to eq $6,(\boldsymbol{M} \cdot \boldsymbol{O})_{H_{\mathrm{v}}}$ and $(\boldsymbol{M} \cdot \boldsymbol{O})_{V_{\mathrm{v}}}$ vary sinusoidally with a spacing $\Lambda$. The spacing is given by eq 6 to 8

$$
\cos 2 \omega=\cos \left(\frac{4 \pi l}{R}\right) r=\cos \left(\frac{2 \pi}{\Lambda} r\right)
$$

where $\Lambda$ is defined as

$$
\Lambda \equiv \frac{1}{2}\left(\frac{R}{l}\right)=P / 2 \equiv \rho
$$

and is equal to one half the pitch $P$ over which the optical axis makes one complete turn. Scattering theory generally predicts that the Fourier transform of a sinusoidally varying induced dipole moment with spacing $\Lambda$ gives only a single scattering maximum at the scattering angle satisfying the Bragg equation $^{12,25,31,32}$

$$
h_{m}=2 \pi / \Lambda \text { or } 2 \Lambda \sin \left(\theta_{m} / 2\right)=\lambda
$$

where $h_{m}$ and $\theta_{m}$ are the scattering vector $\boldsymbol{h}$ and angle at which the ring scattering becomes a maximum,

$$
\boldsymbol{h}=(4 \pi / \lambda) \sin (\theta / 2)
$$

In eq $12, \theta$ and $\lambda$ are the scattering angle and wavelength of the incident light in the medium. The scattering functions predicting eq 11 were obtained by Stein et al. for ringed spherulites $^{12,31}$ and by Hashimoto et al. for cholesteric liquid crystals. ${ }^{25,32}$

Thus, when $\beta=90^{\circ}$, a single scattering maximum should appear at $\theta=\theta_{m}$ and be reciprocally related to one half-pitch $P / 2=\rho$ rather than to $P$ itself. The fact that $\theta_{m}$ depends on $\rho$ rather than $P$ arises from the physical indistinguishability of the vectors $\boldsymbol{a}$ and $-\boldsymbol{a}$ (or of $\omega$ and $\omega+\pi)$ in the case of $\beta=90^{\circ}$. Since the absolute value of induced dipole moment under $H_{\mathrm{v}}$ polarization becomes maxima at odd multiples of $\alpha=45^{\circ}$ (eq 7 and Figure 6a), the $H_{\mathrm{v}}$ scattering has maximum intensities at odd multiples of $\mu=45^{\circ}$ (Figure 6c). The higher- 
Light Scattering and Crystallization of Poly(decamethylene terephthalate)

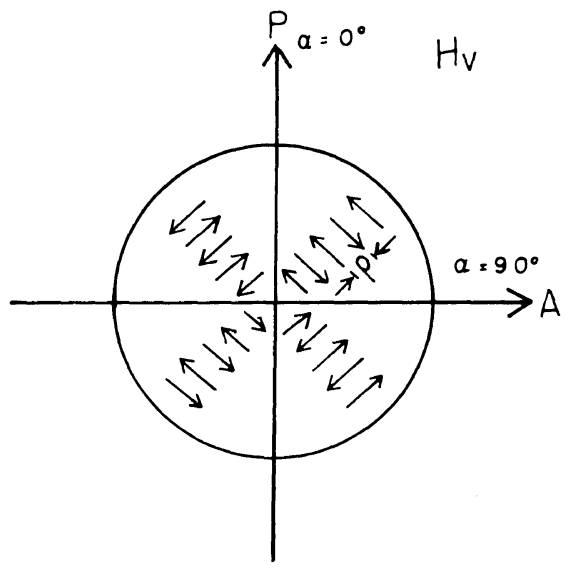

(a)

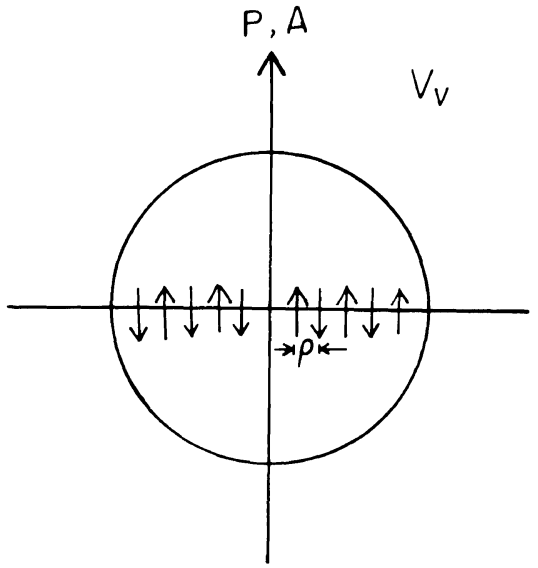

(b)

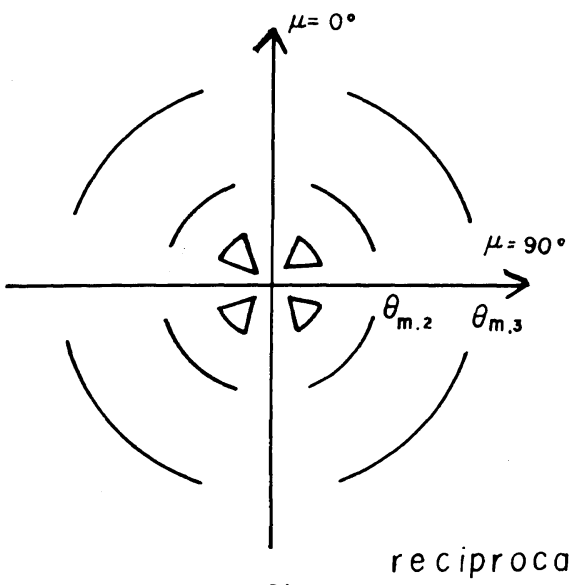

(C)

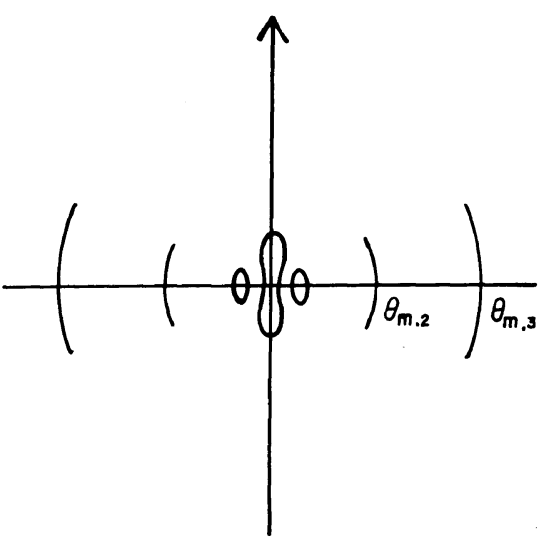

(d)

$$
\begin{aligned}
& \theta_{m_{3} 3} \simeq 2 \theta_{m_{2} 2}, 2 \text { nd-order diffraction of } \Lambda=\rho=P / 2 \\
& \theta_{m_{2} 2} \sim 1 / \rho ; \text { I st-order diffraction of } \Lambda=\rho=P / 2
\end{aligned}
$$

Figure 6. Ringed spherulite with $\beta=90^{\circ}$ in real space (top two figures) and a reciprocal space (bottom two figures) under $H_{\mathrm{v}}$ (left two figures) and $V_{\mathrm{v}}$ polarization conditions (right two figurēs).

order scattering maxima at $\theta=\theta_{m, 3}=2 \theta_{m, 2}$ can be predicted for the case where the optical axis is twisted nonlinearly about the spherulite radius. $^{32}$

$$
2 \rho \sin \left(\theta_{m, n+1} / 2\right)=n \lambda \quad(n=1,2)
$$

Since the induced dipole moment under $V_{v}$ scattering attains a maximum at $\alpha=90^{\circ}$ (eq 8 and Figure $6 \mathrm{~b}$ ), the ring scattering appears at $\mu=90^{\circ}$ (Figure 6d).

When $\beta=45^{\circ}$, the situation is quite different from that for $\beta=90^{\circ}$, as may be intuitively seen from Figure 7; (a) for $H_{\mathrm{v}}$ and (b) for $V_{\mathrm{v}}$ scattering conditions. For example, under the 


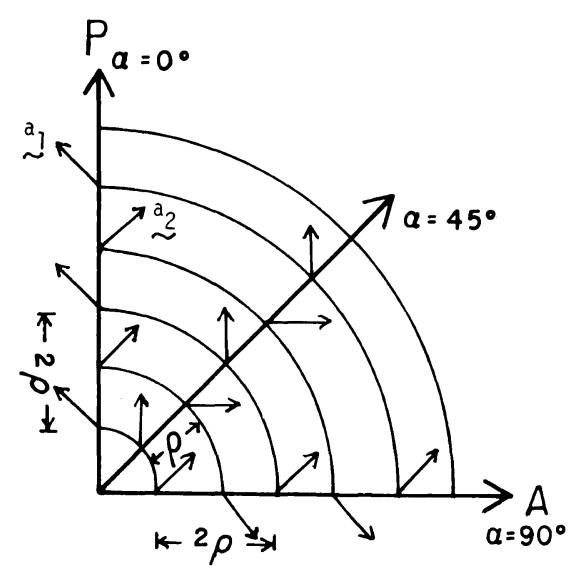

(a)

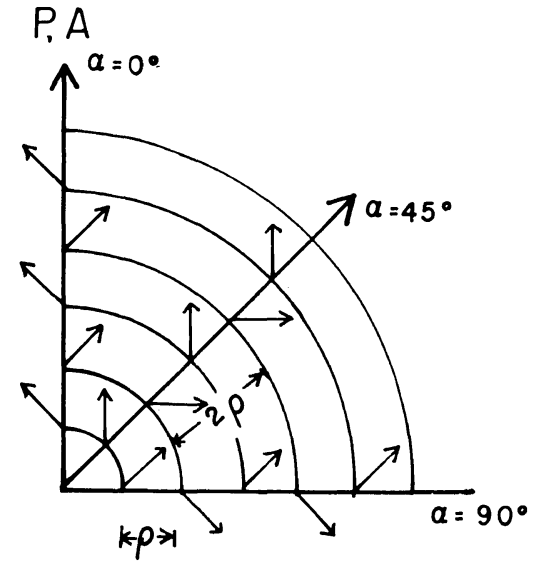

(b)

\section{Hv}

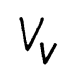

Figure 7. Ringed spherulite with $\beta=45^{\circ}$ in real space (a) $H_{\mathrm{v}}$ and (b) $V_{\mathrm{v}}$ polarization conditions. $\mathbf{a}_{1}$ and $\mathbf{a}_{2}$ designate unit vectors parallel to optical axes.

$H_{\mathrm{v}}$ scattering condition (Figure 7(a)), a part of the spherulite parallel to the polarizer and analyzer axes (i.e., regions of $\alpha=0$ and $90^{\circ}$ ) has induced dipole moments which vary spatially with the spacing $\Lambda=2 \rho=P$, i.e., the pitch of the twisting, while a part of the spherulite at odd multiples of $\alpha=45^{\circ}$ with respect to the polarizer and analyzer axes has induced dipole moments varying with the spacing $\Lambda=\rho=$ (1/2)P.

Under the $V_{\mathrm{v}}$ scattering condition (Figure $7 b$ ), a part of the spherulite parallel to the polarizer and analyzer axes shows no periodic variation in the induced dipole moment at all, although the optical axis itself is twisted with the spacing $\Lambda=2 \rho$. A part of the spherulite at odd multiples of $\alpha=45^{\circ}$ has an induced dipole moment which varies with $\Lambda=2 \rho=P$, while that of the spherulite at either $\alpha=90^{\circ}$ or $180^{\circ}$ has an induced dipole moment which varies with $\Lambda=\rho=P / 2$.

A more quantitative argument can be put forward based on eq 4 to 6 . Thus, for $\beta=45^{\circ}$, the induced dipole moments are given by

$$
\begin{aligned}
& (\boldsymbol{M} \cdot \boldsymbol{O})_{H_{v}, \beta=45^{\circ}} \\
& =-\frac{1}{2} E_{0}\left(\alpha_{1}-\alpha_{2}\right)\left[\frac{1}{4} \cos 2 \omega \sin 2 \alpha\right. \\
& \left.\quad+\cos \omega \cos 2 \alpha-\frac{1}{4} \sin 2 \alpha\right] \\
& (\boldsymbol{M} \cdot \boldsymbol{O})_{V_{v}, \beta=45^{\circ}} \\
& =\frac{1}{2} E_{0}\left(\alpha_{1}-\alpha_{2}\right)\left[\frac{1}{2} \cos 2 \omega \sin ^{2} \alpha\right. \\
& \left.\quad+\frac{1}{2} \cos \omega \sin 2 \alpha+\frac{1}{2}\left(1+\cos ^{2} \alpha\right)\right] \\
& \quad+E_{0} \alpha_{2}
\end{aligned}
$$

The induced dipole moments obviously have components which spatially vary with the spacing $\Lambda=\rho=(1 / 2) P$ (i.e., the term associated with $\cos 2 \omega$ ) and $\Lambda=2 \rho=P$ (i.e., the term associated with $\cos \omega)$. Each component contributes differently to the scattering, since the coefficients of $\cos 2 \omega$ and $\cos \omega$ have different angular dependence with respect to $\alpha$. For example, under the $H_{\mathrm{v}}$ scattering condition, the absolute value of the term $\cos 2 \omega$ has 


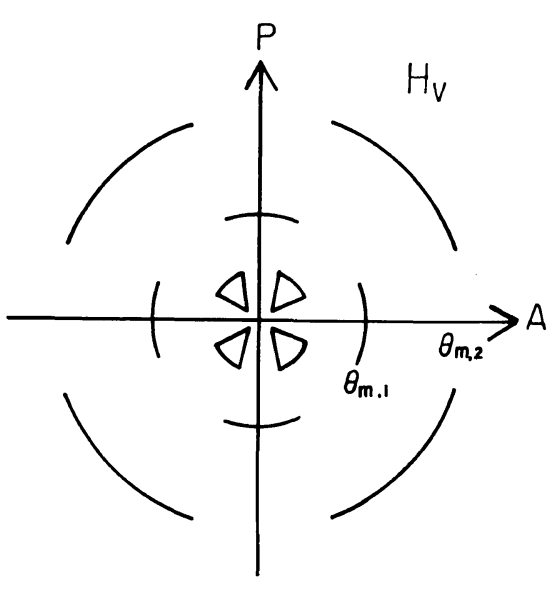

(a)

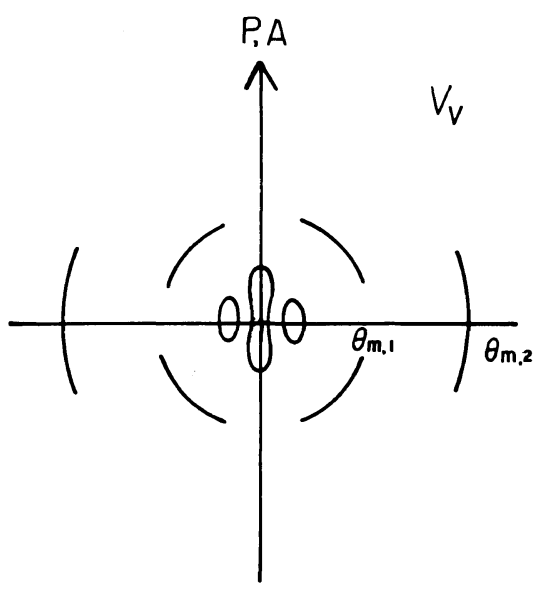

(b)

$$
\begin{aligned}
& \theta_{m, 2} \simeq 2 \theta_{m, 1} \\
& \theta_{m, 1} \sim 1 / P \quad \text { : Ist-order diffraction of } \Lambda=P=2 p \\
& \theta_{m, 2} \sim 2 / P=1 / p \quad \text {; I st- order diffraction of } \Lambda=P / 2=\rho
\end{aligned}
$$

Figure 8. Ringed spherulite with $\beta=45^{\circ}$ in a reciprocal space: (a) $H_{\mathrm{v}}$ and (b) $V_{\mathrm{v}}$ polarization conditions. The second-order diffraction arcs of $\Lambda=\rho$ at $\theta=\theta_{m, 3}$ are not shown in the figure.

maximum values at odd multiples of $\alpha=45^{\circ}$, and hence the corresponding $H_{\mathrm{v}}$ scattering has maximum intensities at odd multiples of $\mu=$ $45^{\circ}$, while that of the term $\cos \omega$ has maximum values at multiples of $\alpha=90^{\circ}$, and hence the corresponding $H_{\mathrm{v}}$ scattering has maximum intensities at odd multiples of $\mu=90^{\circ}$. We note that the term $\cos 2 \omega$ gives a ring scattering maximum at $\theta=\theta_{m, 2}$, while the term $\cos \omega$ gives a maximum at $\theta=\theta_{m, 1}=\theta_{m, 2} / 2$ (eq $9-$ 11). Thus, the $H_{\mathrm{v}}$ scattering should have a pattern such as that schematically shown in Figure 8(a). Similarly, the $V_{\mathrm{v}}$ scattering should have a pattern such as schematically shown in Figure 8(b).

$(\boldsymbol{M} \cdot \boldsymbol{O})_{\boldsymbol{H}_{\mathrm{v}}, \beta=45^{\circ}}$ has a periodicity $f(\omega)=$ $f(\pi+\omega)$ only at odd multiples of $\alpha=45^{\circ}$, showing a spatial variation with $\Lambda=\rho$. Therefore, the $H_{\mathrm{v}}$ scattering at odd multiples of $\mu=45^{\circ}$ becomes a maximum at $\theta=\theta_{m, 2}$. At another values of $\alpha,(\boldsymbol{M} \cdot \boldsymbol{O})_{H_{\mathrm{v}}, \beta=45^{\circ}}$ has a periodicity $f(\omega)=f(2 \pi+\omega)$, showing a spatial variation with $\Lambda=2 \rho=P$. Therefore, the $H_{\mathrm{v}}$ scattering at multiples of $\mu=90^{\circ}$ becomes a maximum at $\theta=\theta_{m, 1}$. A similar argument can be made for the $V_{\mathrm{v}}$ scattering. The predictions leading to Figure 8 may be confirmed by the numerical calculations of Clough, van Aartsen, and Stein (see Figures 5 and 7 of ref 31).

The experimental scattering patterns shown in Figures 3(a) to 3(c) and in Figure 2 (schematic) can, therefore, be interpreted as due to the ringed spherulite with the optical axis inclined at $\beta$ to the spherulite radius, $\beta$ being close to 45 . . The "ring scattering $I$ " may be attributed to the first-order scattering maximum associated with the term $\cos \omega$ (having the spacing $\Lambda=P=2 \rho$ ), and the "ring scattering II and III" to the first and second-order scattering maxima associated with the term $\cos 2 \omega$ (having the spacing $\Lambda=P / 2=\rho$ ) (see Figure 2), respectively. Although there exist a number of apparent spacings $\rho_{\mathrm{I}}$ to $\rho_{\mathrm{III}}$, the optical axes themselves are twisted with a single spacing $P$.

We may propose that the tilting angle $\beta$ can 
be quantitatively estimated by comparing the scattered intensities of rings I and II (Figure 2, eq 4 and 5). The existence of the higher-order ring scattering maximum III is due to nonlinear twisting of the optical axis about the spherulite radius. ${ }^{32}$ It still remains unclear as to why the expected second-order scattering maxima at $\theta=\theta_{m, 2}$ and at multiples of $\mu=90^{\circ}$ corresponding to the ring $I$ in Figure 2 could not be experimentally observed. Probably, the intensity of the second-order maximum was too weak to be measured, as is conceivable from the fairly weak intensity of the first-order maximum of ring I. The absence of the secondorder maximum also should be related to the tilting angle $\beta$.

\section{REFERENCES}

1. P. H. Geil, "Polymer Single Crystal," John Wiley and Sons, New York, N. Y., 1963.

2. R. S. Stein, "Newer Methods of Polymer Characterization," B. Ke Ed., John Wiley and Sons, New York, N. Y., 1964.

3. M. Motegi, T. Oda, M. Moritani, and H. Kawai, Polym. J., 1, 209 (1970).

4. T. Hashimoto, A. Todo, and H. Kawai, J. Polym. Sci., Polym. Phys. Ed., 11, 149 (1973); There are following typographical errors in this article. (1) The last term of right-hand side of eq 17

should be read as $(\cos \beta \cos \alpha+\sin \beta \underline{\cos \Phi} \sin \alpha) k$

(2) The term $(\cos \beta \cos \alpha+\sin \beta \underline{\cos \omega} \sin \alpha) \boldsymbol{k}$.

$\sin \beta \cos \omega \cos \omega \sin ^{2} \alpha$

in the right-hand side of eq 21 should be read as $\sin \beta \cos \beta \cos \omega \sin ^{2} \alpha$.

(3) The term

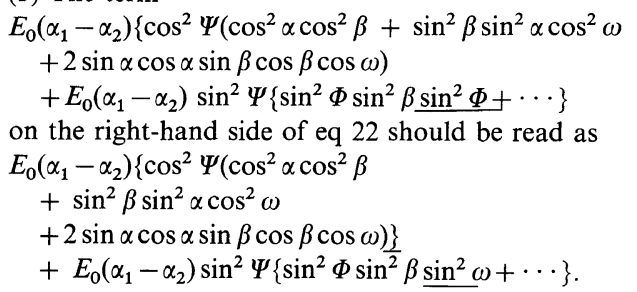

5. M. B. Rhodes and R. S. Stein, J. Polym. Sci., Polym. Lett., 1, 663 (1963).

6. M. B. Rhodes and R. S. Stein, J. Polym. Sci., 62, S84 (1962).

7. I. Daniewska, Polym. J., 12, 417 (1980).

8. Ju. K. Godowskij, Vysokomol. Soedin., Ser. A, 11, 2129 (1969).

9. R. F. Boyer, J. Macromol. Sci., 8, 503 (1973).

10. R. G. Beaman, J. Polym. Sci., 9, 472 (1952).

11. W. A. Lee and G. J. Knight, Br. Polym. J., 2, 73 (1970).

12. R. S. Stein and M. B. Rhodes, J. Appl. Phys., 31, 1873 (1960).

13. R. S. Moore, J. Polym. Sci., A, 3, 4093 (1965).

14. R. S. Stein and A. Plaza, J. Polym. Sci., 45, 146 (1960).

15. P. H. Lindenmeyer and V. L. Holland, J. Appl. Phys., 35, 55 (1964).

16. A. Keller, J. Polym. Sci., 17, 291 (1955).

17. A. Keller, J. Polym. Sci., 39, 151 (1959).

18. R. E. Prud'homme, L. Bourland, R. T. Natarjan, and R. S. Stein, J. Polym. Sci., Polym. Phys. Ed., 12, 1955 (1974).

19. R. T. Natarjan, R. E. Prud'homme, L. Bourland, and R. S. Stein, J. Polym. Sci., Polym. Phys. Ed., 14, 1541 (1976).

20. G. C. Adams and R. S. Stein, J. Polym. Sci. A-2, 6, 31 (1968).

21. T. Hashimoto, Y. Murakami, Y. Okamori, and H. Kawai, Polym. J., 6, 554 (1974).

22. W. G. Baranov and T. P. Volkov, Vysokomol. Soedin. Ser. B, 10, 222 (1968).

23. M. B. Rhodes and R. S. Stein, J. Polym. Sci., Polym. Lett. Ed., 1, 663 (1963).

24. H. D. Keith and F. J. Padden, J. Polym. Sci., 31, 415 (1958).

25. T. Hashimoto, S. Ebisu, N. Inaba, and H. Kawai, Polym. J., 13, 701 (1981).

26. R. S. Moore and Ch. Gieniewski, J. Appl. Phys., 36, 3022 (1965).

27. H. D. Keith and F. J. Padden, J. Polym. Sci., 34, 101 (1959).

28. R. F. Fryer, J. Appl. Sci., 18, 2261 (1974).

29. R. S. Moore and Ch. Gieniewski, J. Polym. Sci., C, 13, 95 (1966).

30. M. B. Rhodes and R. S. Stein, J. Appl. Phys., 39, 4903 (1968).

31. S. B. Clough, J. J. van Aartsen, and R. S. Stein, J. Appl. Phys., 36, 3072 (1965).

32. T. Hashimoto, N. Inaba, S. Ebisu, and H. Kawai, Polym. J., 13, 897 (1981). 